\title{
The role of cerebral hyperperfusion in postoperative neurologic dysfunction after left ventricular assist device implantation for end-stage heart failure
}

Katherine Lietz, MD, PhD, ${ }^{\mathrm{a}}$ Kevin Brown, DO,${ }^{\mathrm{f}}$ Syed S. Ali, MD, ${ }^{\mathrm{c}}$ Monica Colvin-Adams, MD, ${ }^{\mathrm{d}}$ Andrew J. Boyle, MD, ${ }^{\mathrm{d}}$ David Anderson, MD, ${ }^{\mathrm{f}}$ Alan D. Weinberg, MS, ${ }^{\mathrm{c}}$ Leslie W. Miller, MD, ${ }^{\mathrm{g}}$ Soon Park, MD, ${ }^{\mathrm{h}}$ Ranjit John, MD, ${ }^{\mathrm{e}}$ and Ronald M. Lazar, $\mathrm{PhD}^{\mathrm{b}}$

Objective: Cerebral hyperperfusion is a life-threatening syndrome that can occur in patients with chronically hypoperfused cerebral vasculature whose normal cerebral circulation was re-established after carotid endarterectomy or angioplasty. We sought to determine whether the abrupt restoration of perfusion to the brain after left ventricular assist device (LVAD) implantation produced similar syndromes.

\begin{abstract}
Methods: We studied the role of increased systemic flow after LVAD implantation on neurologic dysfunction in 69 consecutive HeartMate XVE LVAD (Thoratec, Pleasanton, Calif) recipients from October 2001 through June 2006. Neurologic dysfunction was defined as postoperative permanent or transient central change in neurologic status, including confusion, focal neurologic deficits, visual changes, seizures, or coma for more than 24 hours within 30 days after LVAD implantation.
\end{abstract}

Results: We found that $19(27.5 \%)$ patients had neurologic dysfunction, including encephalopathy $(\mathrm{n}=11)$, coma $(n=3)$, and other complications $(n=5)$. The multivariate analysis showed that an increase in cardiac index from the preoperative baseline value (relative risk, 1.33 per $25 \%$ cardiac index increase; $P=.01$ ) and a previous coronary bypass operation (relative risk, $4.53 ; P=.02$ ) were the only independent predictors of neurologic dysfunction. Reduction of left ventricular assist device flow in 16 of the 19 symptomatic patients led to improvement of symptoms in $14(87 \%)$ patients.

Conclusions: Our findings showed that normal flow might overwhelm cerebral autoregulation in patients with severe heart failure, suggesting that cerebral hyperperfusion is possible in recipients of mechanical circulatory support with neurologic dysfunction.

Left ventricular assist devices (LVADs) have been successfully used in patients with end-stage heart failure, either as a bridge to transplantation or as an alternative to transplantation or destination therapy. Although LVAD implantation has become an accepted therapy for patients with advanced heart failure, the morbidities associated with use of the device have been an ongoing concern. In particular, postoperative neurologic complications are relatively common, and sometimes they can lead to devastating complications that preclude a transplantation and result in very poor quality of life.

According to the 2005 Report of the Mechanical Circulatory Support Device Database, serious neurologic complica-

From the Center for Advanced Cardiac Care, ${ }^{a}$ the Department of Neurology, ${ }^{\mathrm{b}}$ and the Division of Biostatistics, ${ }^{\mathrm{c}}$ Columbia University Medical Center, New York, NY; the Divisions of Cardiology ${ }^{\mathrm{d}}$ and Cardiothoracic Surgery ${ }^{\mathrm{e}}$ and the Department of Neurology, ${ }^{\mathrm{f}}$ University of Minnesota, Minneapolis, Minn; the Division of Cardiology, ${ }^{\mathrm{g}}$ Georgetown University-Washington Hospital Center, Washington, DC; and the Division of Cardiothoracic Surgery, ${ }^{\text {h }}$ Mayo Clinic, Rochester, Minn.

Received for publication March 12, 2008; revisions received Oct 19, 2008; accepted for publication Nov 22, 2008.

Address for reprints: Katherine Lietz, MD, PhD, Center for Advanced Cardiac Care, Division of Cardiology, Columbia University Medical Center, PH-12 Stem Rm 134, 622W 168th St, New York, NY 10032 (E-mail: KL2384@columbia.edu).

J Thorac Cardiovasc Surg 2009; 137:1012-9

$0022-5223 / \$ 36.00$

Copyright (c) 2009 by The American Association for Thoracic Surgery doi:10.1016/j.jtcvs.2008.11.034 tions have been reported in $14 \%$ of 655 recipients with various types of mechanical circulatory support pumps implanted at 60 international centers. ${ }^{1}$ In LVAD recipients the reported incidence of postoperative neurologic complications varies by center and pump type, as described in detail by Pae and colleagues. ${ }^{2}$ In the largest published single-center studies, the stroke incidence ranges from $3 \%$ in 226 HeartMate XVE LVAD (Thoratec, Pleasanton, Calif) recipients $^{3}$ and $17 \%$ in 288 Novacor LVAD (WorldHeart, Oakland, Calif) recipients ${ }^{4}$ to as high as $57 \%$ in 23 recipients of the totally implantable LionHeart LVAD (Arrow International, Inc, Reading, Pa). ${ }^{2}$

Most postoperative neurologic complications, including the most serious events, such as strokes, occur within the first few days or weeks after LVAD implantation. ${ }^{1-4}$ Hence the incidence of perioperative neurologic complications after LVAD surgery exceeds by several times the relatively small 1.9\% incidence noted after other cardiac operations, ${ }^{5}$ suggesting an association between the development of neurologic complications and either the device itself or the operation needed to implant it. The exact mechanism by which LVAD implantation contributes to the development of symptoms is not completely understood. Most of the literature assumes that clinically significant neurologic events result either from device-related thromboembolism, ${ }^{6}$ 

Abbreviations and Acronyms
$\begin{array}{ll}\mathrm{CI} & =\text { cardiac index } \\ \mathrm{CT} & =\text { computed tomographic }\end{array}$
IABP $=$ intra-aortic balloon pump
LVAD $=$ left ventricular assist device
$\mathrm{ND}=$ neurologic dysfunction
PRES = posterior reversible encephalopathy syndrome
$\mathrm{RR} \quad=$ relative risk

operation-related air embolism, ${ }^{7}$ or hemorrhage. ${ }^{8}$ Autopsies of patients with fatal neurologic complications, however, did not always reveal evidence of cerebral embolism. ${ }^{6,7}$

In this retrospective review we investigated whether an abrupt increase in systemic blood flow could contribute to LVAD-related neurologic dysfunction (ND) in patients with end-stage heart failure. We hypothesized that restoration of normal cardiac output in this patient group might result in cerebral dysfunction, which is similar to the cerebral hyperperfusion syndrome observed in some patients after carotid artery endarterectomy or angioplasty. ${ }^{9}$ This syndrome is believed to result from the inability of chronically hypoperfused cerebral arterioles to accommodate rapidly increasing perfusion pressures.

\section{MATERIALS AND METHODS Patients}

From October 30, 2001, through June 13, 2006, a total of 69 patients underwent HeartMate XVE LVAD implantation at the University of Minnesota Medical Center. We excluded from our analysis recipients of other types of HeartMate LVADs, Novacor LVADs, and axial flow devices, as well as those who required biventricular temporary support before device implantation. The institutional board review approved the study, and all patients provided informed consent to use their clinical data for this study.

\section{Study Design}

Because of the possibility of delayed impairment of cerebral autoregulation up to 28 days after increase of cerebral flow reported after carotid endarterectomy, ${ }^{10}$ in this study we investigated the relationship between systemic blood flow and ND within the first month after LVAD implantation.

Preoperative clinical data. The following clinical data were available on the day of the operation: patient characteristics and body surface area; cause of heart failure; history of cardiovascular events (including cardiac arrest, myocardial infarction, previous sternotomies, and coronary artery bypass surgery); history of other comorbidities (including stroke, diabetes, or renal dysfunction); laboratory data; left ventricular ejection fraction; and details on medical or device therapy for heart failure (including the use of intravenous inotropes, mechanical circulatory support with an intraaortic counterpulsation balloon pump [IABP] and mechanical ventilation).

Neurologic dysfunction. We defined perioperative neurologic dysfunction (ND) as a change in neurologic status, including confusion, focal neurologic deficits, visual changes, seizures, or coma, for more than 24 hours within 30 days after LVAD implantation, which is consistent with previously described criteria of cerebral hyperperfusion syndrome. ${ }^{9}$

Hemodynamic changes. Preoperative cardiac output was measured on the day of hospital admission and on the day of LVAD surgery with the use of a Swan-Ganz catheter and was calculated from the Fick equation. After LVAD surgery, the Swan-Ganz catheter was removed. Cardiac output was recorded from the flow displayed on the device monitor at the time of the neurologic event, on the third postoperative day, and on postoperative day 30 or day of hospital discharge, whichever came first. The cardiac index after LVAD implantation was calculated by dividing the device-recorded flow (liters per minute) by the patient's body surface area.

\section{LVAD Surgery}

All recipients of LVAD as a bridge to transplantation underwent a complete workup to assess their transplantation candidacy, including carotid and lower-extremity arterial Doppler studies, pulmonary function tests, colonoscopy, a neuropsychiatric evaluation, and an evaluation by a social worker. Patients whose LVADs were intended as destination therapy underwent the same workup. History of stroke or neurologic injury was not a contraindication to LVAD implantation as long as the patient had recovered sufficiently from the neurologic injury, the underlying cause had been corrected, and the risks for stroke recurrence were low. All studied patients had carotid Doppler ultrasonographic analysis performed before LVAD insertion or, in case of emergency implantation, after LVAD insertion to minimize significant intracranial or extracranial cerebral disease. Also, head computed tomographic (CT) analysis was performed in 4 patients who had previous strokes.

LVAD implantation was performed after achievement of general anesthesia, with continuous hemodynamic monitoring. Either preperitoneal or intraperitoneal LVAD implantation was performed through a medial sternotomy. Cardiopulmonary bypass was instituted after full heparinization by means of aortic and right atrial cannulation. Only mild hypothermia was used during the operation $\left(>32^{\circ} \mathrm{C}\right)$. The LVAD inflow cannula was secured to the left ventricular apex, and the outflow graft was anastomosed to the ascending aorta. The driveline of the LVAD was usually tunneled out through a right upper quadrant incision. Intraoperative transesophageal echocardiographic analysis was used to confirm the evacuation of air from the cardiac chambers. The operative field was flooded by carbon dioxide to reduce the collection of air inside the heart. Standard maneuvers to evacuate air included filling the heart with blood and ventilating the lungs, along with hand pumping of the LVAD. As the patient was weaned off cardiopulmonary bypass, the LVAD pump was activated with the patient in a steep Trendelenburg position to reduce the risk of cranial air embolism. Once the patient was hemodynamically stable with adequate LVAD flow, protamine was used for reversal of heparin. Appropriate inotropes and vasopressors were administered, as necessary, to maintain hemodynamic stability and to optimize right ventricular function. Postoperatively, the only routine antithrombotic therapy was aspirin $(325 \mathrm{mg} / \mathrm{d})$. Warfarin was used only if there were indications for its use, such as pre-existing atrial fibrillation or the presence of a mitral mechanical valve prosthesis.

\section{Statistical Analysis}

Preoperative clinical data and hemodynamic changes before and after LVAD implantation were associated with the end point by using univariate analysis to identify risk factors associated with perioperative ND in LVAD recipients. In patients with $\mathrm{ND}$, we compared the hemodynamic measurements at the time of the neurologic event with those of patients who did not have ND by postoperative day 30 or the day of hospital discharge, whichever came first. All continuous variables were treated as such, with the exception of recipient age and body surface area, which were stratified by their means. The risk factors found to be significant by means of univariate analysis at a $P$ value of less than .10 were then entered into the logistic regression model at a $P$ value of less than $.05 .{ }^{10}$ The figure showing a relationship between the increase in cardiac index $(\mathrm{CI})$ and the predicted probability of postoperative ND was derived from the logistic regression model. The goodness of fit of this model was measured with the Hosmer-Lemeshow statistic. ${ }^{11}$ Differences between groups were examined by using $\chi^{2}$ or Student's $t$ tests. Values are reported as means \pm the standard deviation. 
TABLE 1. Baseline patient characteristics $(n=69)$

\begin{tabular}{lc}
\hline Male sex, $\mathrm{n}(\%)$ & $59(85.5)$ \\
Age $(\mathrm{y})$, mean $\pm \mathrm{SD}$ & $54.3 \pm 10.7$ \\
White, $\mathrm{n}(\%)$ & $65(94.2)$ \\
Body surface area $\leq 2 \mathrm{~m}^{2}, \mathrm{n}(\%)$ & $21(30.4)$ \\
Medical history & \\
Ischemic cardiomyopathy, $\mathrm{n}(\%)$ & $40(58.0)$ \\
Myocardial infarction, $\mathrm{n}(\%)$ & $26(37.7)$ \\
Cardiac arrest, $\mathrm{n}(\%)$ & $12(17.4)$ \\
Sternotomy, $\mathrm{n}(\%)$ & $24(34.8)$ \\
Coronary artery bypass surgery, $\mathrm{n}(\%)$ & $22(31.9)$ \\
Diabetes mellitus, $\mathrm{n}(\%)$ & $25(36.2)$ \\
Renal disease, $\mathrm{n}(\%)$ & $12(17.4)$ \\
Stroke, $\mathrm{n}(\%)$ & $4(5.8)$ \\
Laboratory results & \\
International normalized ratio, mean $\pm \mathrm{SD}$ & $1.32 \pm 0.28$ \\
White blood count $\left(\times 10^{3} / \mu \mathrm{L}\right)$, mean $\pm \mathrm{SD}$ & $9.1 \pm 3.0$ \\
Hemoglobin $(\mathrm{mg} / \mathrm{dL})$, mean $\pm \mathrm{SD}$ & $11.9 \pm 1.8$ \\
Platelet count $\left(\times 10^{3} / \mu \mathrm{L}\right), \mathrm{mean} \pm \mathrm{SD}$ & $217.9 \pm 66.7$ \\
Serum sodium $(\mathrm{mg} / \mathrm{dL})$, mean $\pm \mathrm{SD}$ & $135.3 \pm 4.3$ \\
Blood urea nitrogen $(\mathrm{mg} / \mathrm{dL})$, mean $\pm \mathrm{SD}$ & $35.8 \pm 22.4$ \\
Serum creatinine $(\mathrm{mg} / \mathrm{dL})$, mean $\pm \mathrm{SD}$ & $1.5 \pm 0.6$ \\
Total bilirubin $(\mathrm{mg} / \mathrm{dL})$, mean $\pm \mathrm{SD}$ & $1.5 \pm 1.2$ \\
Albumin $(\mathrm{g} / \mathrm{mL}), \mathrm{mean} \pm \mathrm{SD}$ & $3.4 \pm 0.7$ \\
Aspartate aminotransferase $(\mathrm{U} / \mathrm{mL}), \mathrm{mean} \pm \mathrm{SD}$ & $162.2 \pm 428.5$ \\
Alanine aminotransferase $(\mathrm{U} / \mathrm{mL}), \mathrm{mean} \pm \mathrm{SD}$ & $141.1 \pm 353.5$ \\
\hline$S D$, &
\end{tabular}

SD, Standard deviation.

Data were analyzed with the SAS System (version 9.0; SAS Institute, Inc, Cary, NC).

\section{RESULTS \\ Patient Characteristics}

The baseline characteristics and hemodynamic severity of heart failure for 69 patients (Tables 1 and 2) were obtained on the day of admission for LVAD implantation. Most patients were male $(85 \%)$, were older than 50 years (71\%), and had ischemic cardiomyopathy $(58 \%)$. All patients had severe heart failure, as evidenced by a mean left ventricular ejection fraction of $17.0 \% \pm 6.3 \%$, systolic blood pressure of $95.7 \pm 11 \mathrm{~mm} \mathrm{Hg}$, cardiac index of $1.73 \pm$ $0.4 \mathrm{~L} \cdot \mathrm{min}^{-1} \cdot \mathrm{kg}^{-2}$, and pulmonary capillary wedge pressure of $23.8 \pm 6.3 \mathrm{~mm} \mathrm{Hg}$. On the day of admission, all patients were maintained on continuous intravenous inotropic support, 6 of whom were supported with an IABP. After the decision was made to proceed with LVAD implantation, we electively placed the IABP the day before the operation in another 58 patients, so that on the day of LVAD implantation, $64(92.7 \%)$ patients were supported with an IABP (mean IABP support time, 1.6 days; range, 12 hours to 7 days).

\section{LVAD Implantation}

LVAD implantation was performed on an emergency basis in $10(14.5 \%)$ patients who were in cardiogenic shock; the remaining 59 patients were scheduled for elective or
TABLE 2. Hemodynamic severity of heart failure on the day of admission $(n=69)$

\begin{tabular}{ll}
\hline Left ventricular ejection fraction $(\%)$, mean $\pm \mathrm{SD}$ & $17.0 \pm 6.3$ \\
Systolic blood pressure $(\mathrm{mm} \mathrm{Hg})$, mean $\pm \mathrm{SD}$ & $95.7 \pm 11.0$ \\
Mean arterial pressure $(\mathrm{mm} \mathrm{Hg})$, mean $\pm \mathrm{SD}$ & $70.4 \pm 10.6$ \\
Heart rate (beats/min), mean $\pm \mathrm{SD}$ & $92.8 \pm 14.3$ \\
Stroke volume $(\mathrm{mL})$, mean $\pm \mathrm{SD}$ & $39.6 \pm 12.6$ \\
Cardiac output $(\mathrm{L} / \mathrm{min})$, mean $\pm \mathrm{SD}$ & $3.59 \pm 0.96$ \\
Cardiac index $\left(\mathrm{L} \cdot \mathrm{min}^{-1} \cdot \mathrm{m}^{-2}\right)$, mean $\pm \mathrm{SD}$ & $1.73 \pm 0.4$ \\
Systolic pulmonary pressure $(\mathrm{mm} \mathrm{Hg})$, mean $\pm \mathrm{SD}$ & $51.7 \pm 13.3$ \\
Pulmonary capillary wedge pressure $(\mathrm{mm} \mathrm{Hg})$, mean $\pm \mathrm{SD}$ & $23.8 \pm 6.3$ \\
Central venous pressure $\left(\mathrm{cm} \mathrm{H}_{2} \mathrm{O}\right)$, mean $\pm \mathrm{SD}$ & $14.2 \pm 5.3$ \\
\hline
\end{tabular}

$S D$, Standard deviation.

semielective surgical intervention. At the time of LVAD implantation, coronary artery bypass surgery was performed in 16 patients, and a patent foramen ovale was closed in 7 patients. The mean cardiopulmonary bypass time was 154.4 minutes (range, 89-323 minutes).

\section{0-Day Outcomes of LVAD Surgery}

Survival and hospital discharge. For the purpose of this analysis, all 69 patients were followed from the day of LVAD implantation until postoperative day 30 or the day of hospital discharge, whichever came first, for a mean follow-up of 23.5 days (range, 6-31 days). During the follow-up period, $35(51 \%)$ patients were discharged home, $26(38 \%)$ remained hospitalized, $5(7 \%)$ underwent a heart transplantation, $2(3 \%)$ died, and $1(1 \%)$ underwent LVAD explantation. Six $(8.7 \%)$ patients required implantation of a right ventricular assist device after LVAD implantation.

Cardiac index after LVAD implantation. The mean CI increased from $1.73 \pm 0.4 \mathrm{~L} \cdot \mathrm{min}^{-1} \cdot \mathrm{m}^{-2}$ on the day of admission to $2.3 \pm 0.3 \mathrm{~L} \cdot \mathrm{min}^{-1} \cdot \mathrm{m}^{-2}$ on the third postoperative day and to $2.36 \pm 0.3 \mathrm{~L} \cdot \mathrm{min}^{-1} \cdot \mathrm{m}^{-2}$ on postoperative day 30 or the day of hospital discharge. In $42 \%$ of LVAD recipients, $\mathrm{CI}$ increased by more than $50 \%$.

\section{Patients With Versus Without ND}

Of the 69 studied patients, $19(27.5 \%)$ had ND postoperatively, and in $13(68 \%)$ of these patients, ND occurred within 2 weeks of surgical intervention (mean time to the onset of symptoms was 12 days), as shown in Table 3 .

Baseline characteristics and severity of heart failure. Patients with ND were more likely to be older than 50 years $(89.5 \%$ with ND vs $64 \%$ without ND, $P=.05)$ and to have undergone a previous sternotomy (52.6\% with ND vs $28 \%$ without ND, $P=.09)$. Of note, $22(92 \%)$ of 24 of patients with previous sternotomies underwent coronary artery bypass surgery. There were no significant differences between the 2 groups (with vs without ND) in terms of patient sex, history of cardiovascular disease, comorbidities, or laboratory markers of renal and hepatic function and of coagulation 
TABLE 3. Clinical symptoms, cardiac index, and computed tomographic results in LVAD recipients with neurologic dysfunction $(\mathrm{n}=19)$

\begin{tabular}{|c|c|c|c|c|c|c|c|}
\hline \multirow{2}{*}{$\begin{array}{l}\text { Patient } \\
\text { no. }\end{array}$} & \multirow{2}{*}{$\begin{array}{c}\text { Postoperative } \\
\text { day }\end{array}$} & \multirow[b]{2}{*}{ Symptoms } & \multirow{2}{*}{$\begin{array}{c}\text { Loss of } \\
\text { consciousness }\end{array}$} & \multirow{2}{*}{$\begin{array}{c}\text { Computed tomography } \\
\text { of head } \\
\end{array}$} & \multicolumn{2}{|c|}{$\begin{array}{c}\text { Cardiac index, } L \cdot \min ^{-1} \cdot \mathbf{m}^{-2} \\
(\% \text { increase from baseline })\end{array}$} & \multirow{2}{*}{$\begin{array}{c}\text { Improvement of } \\
\text { symtoms }\end{array}$} \\
\hline & & & & & At time of event & After event & \\
\hline 1 & 10 & Coma & Yes & Ventricular hemorrhage & $1.91(19.4)$ & $1.91(19.7)$ & No \\
\hline 2 & 3 & Coma, seizure & Yes & Left frontal infarct & $2.89(70.4)$ & $1.91(12.5)$ & No \\
\hline 3 & 30 & Left hemiparesis & Yes & Negative & $2.75(83.4)$ & $2.25(49.8)$ & Yes \\
\hline 4 & 17 & Left hemiparesis & Yes & Negative & $2.90(107)$ & $2.50(78.6)$ & Yes \\
\hline 5 & 3 & Left hemiplegia & Yes & Negative & $3.02(77.7)$ & $2.24(31.7)$ & Yes \\
\hline 6 & 12 & Left hemiparesis & No & Negative & $2.86(50.4)$ & $1.97(3.7)$ & Yes \\
\hline 7 & 25 & Encephalopathy & No & Not done & $2.89(60.5)$ & $2.44(35.8)$ & Yes \\
\hline 8 & 25 & Encephalopathy & No & Not done & $2.80(86.7)$ & $2.50(66.7)$ & No \\
\hline 9 & 8 & Confusion & No & Not done & $2.92(94.7)$ & $2.22(47.7)$ & Yes \\
\hline 10 & 6 & Confusion & No & Not done & $3.42(184.9)$ & $2.52(110.8)$ & Yes \\
\hline 11 & 16 & Confusion & No & Negative & $3.20(100.2)$ & $2.72(69.9)$ & Yes \\
\hline 12 & 26 & Confusion & No & Not done & $2.39(32.5)$ & $2.74(52.3)$ & Yes \\
\hline 13 & 7 & Confusion & No & Negative & $2.48(147.8)$ & $1.95(94.7)$ & Yes \\
\hline 14 & 11 & Confusion & No & Not done & $3.72(96.1)$ & $2.40(26.4)$ & Yes \\
\hline 15 & 1 & Agitation & No & Negative & $2.59(99.2)$ & $2.31(78.1)$ & Yes \\
\hline 16 & 10 & Confusion, seizure & No & Negative & $2.68(57.4)$ & $2.67(57.4)$ & Yes \\
\hline 17 & 1 & Seizure & No & Negative & $2.18(26.7)$ & $2.01(17.2)$ & Yes \\
\hline 18 & 6 & $\begin{array}{l}\text { Partial vision loss and } \\
\text { confusion }\end{array}$ & No & Negative & $3.04(32.2)$ & $2.44(6.3)$ & Yes \\
\hline 19 & 8 & Loss of vision in left eye & No & Negative & $2.81(75.7)$ & $2.59(62.2)$ & Yes \\
\hline
\end{tabular}

abnormalities. We also found no significant differences in terms of hemodynamic measures of severity of heart failure.

Hemodynamic measures. Increases in CI, stroke volume, and mean arterial pressure after LVAD implantation were stratified by development of ND in Figure 1. There were no significant differences between the groups (with vs without ND) except at the time of the neurologic event: CIs were significantly higher in patients with ND compared with those in control subjects assessed on postoperative day 30 or the day of hospital discharge (mean CI, $2.8 \mathrm{~L} \cdot \min ^{-1}$. $\mathrm{m}^{-2}$ with ND vs $2.4 \mathrm{~L} \cdot \min ^{-1} \cdot \mathrm{m}^{-2}$ without ND; $P=$ .002). The LVAD settings at the time of the event were more likely to be set in an automatic mode in patients with $\mathrm{ND}(52.6 \%$ in patients with ND vs $18 \%$ in patients without ND, $P=.004)$. CI increase of more than $50 \%$ from the preoperative baseline value was found in $15(79 \%)$ of 19 patients with ND.

\section{Risk Factors for ND}

Using logistic regression analysis, we identified 2 independent predictors of postoperative ND: an increase of CI from preoperative baseline values (relative risk [RR], 1.33; [95\% confidence interval, $0.99-1.78$ ] per $25 \%$ increase of $\mathrm{CI}, P=.01)$ and history of coronary bypass surgery (RR, 4.53 [95\% confidence interval, $1.4-14.7] ; P=.02$ ). The Hosmer-Lemeshow goodness-of-fit test indicated that the logistic regression model was a good fit $(P=.90)$. The cor- relation between the predicted probability of ND after LVAD implantation and the increase in CI derived from the above model is shown in Figure 2. There was a direct relationship between the risk of ND and increase in CI in all studied patients, although those who previously underwent coronary bypass surgery appeared to be at an overall higher risk. The correlation between the observed incidence of ND in the studied cohort and the increase of CI is shown in Figure 3.

Risk factors found significant by means of univariate analysis at a $P$ value of less than .05 that did not enter the final model included patient age of greater than 50 years (RR, 4.8; $P=.04$ ) and body surface area of $2 \mathrm{~m}^{2}$ or less (RR, 2.8; $P=.06)$. No other parameters correlated by means of univariate analysis with ND, including patient sex; cause of heart failure; history of myocardial infarction or cardiac arrest; history of other comorbidities, including stroke, diabetes, or renal dysfunction; emergency LVAD implantation; measures of hemodynamic severity of heart failure at the time of admission and on the day of the operation; preoperative laboratory data; cardiopulmonary bypass time; or device mode or rate.

\section{Reduction in CI and Recovery From ND}

Development of ND prompted reduction of LVAD flow in $16(84 \%)$ of the 19 patients (Table 3 and Figure 4$)$. The mean CI was reduced from $2.81 \pm 0.41$ to $2.33 \pm 0.28 \mathrm{~L}$ $\cdot \min ^{-1} \cdot \mathrm{m}^{-2}(P<.001)$. The LVAD settings were changed to fixed mode in 11 patients with ND whose settings were in 

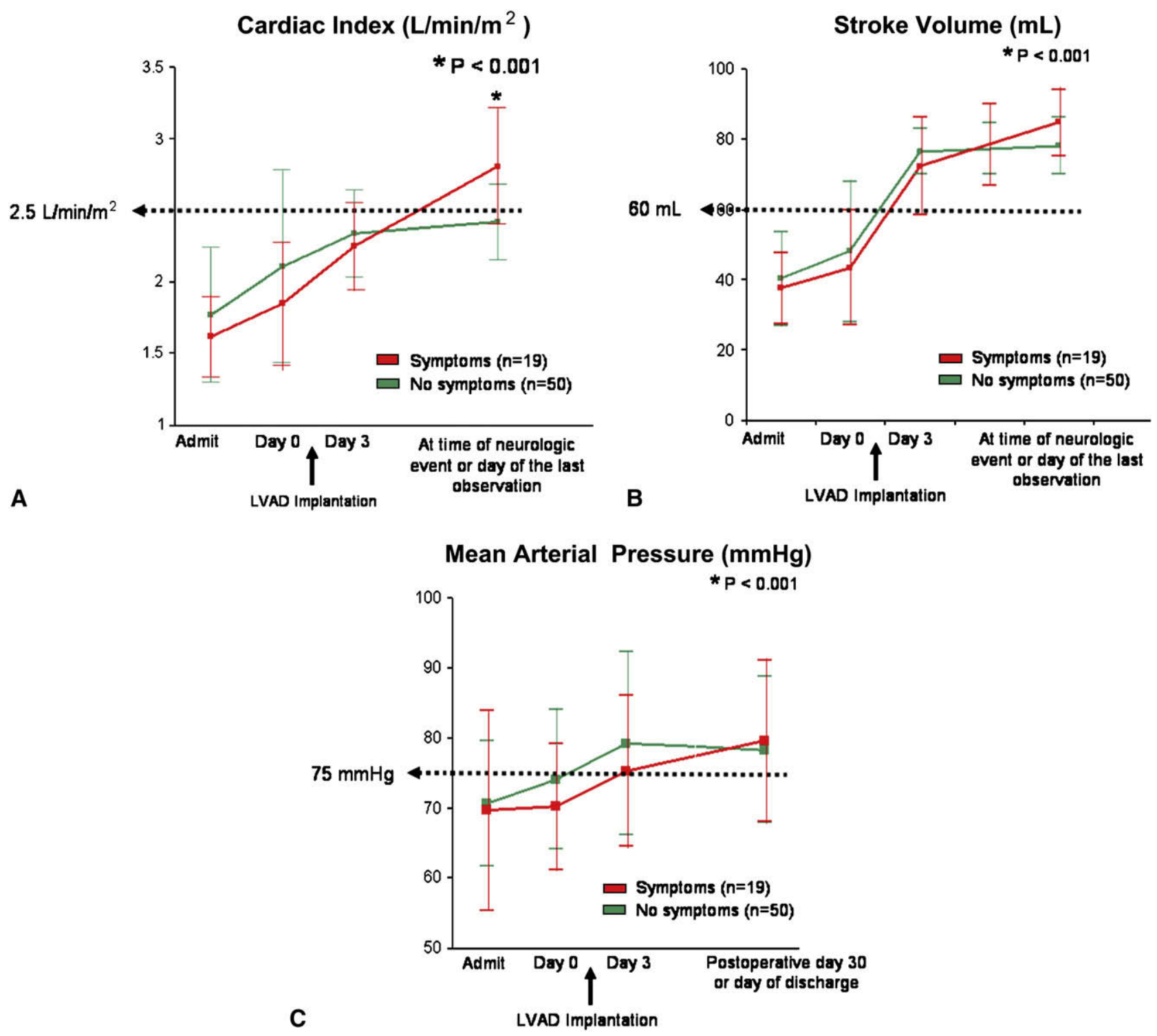

FIGURE 1. Comparisons of cardiac index (A), stroke volume (B), and mean arterial pressure (C) in patients with and without neurologic dysfunction within 30 days after left ventricular assist device (LVAD) implantation are shown. Hemodynamics were assessed on the day of admission, the day of LVAD surgery, postoperative day 3 , and postoperative day 30 or the day of hospital discharge, whichever came first. Asterisks indicate statistical difference between patients with and without neurologic dysfunction.

automatic mode at the time of their neurologic event. Reduced LVAD flow and CI correlated with the improvement of ND (Figure 4), with clinical improvement or resolution by 48 hours and without negative consequences to patients. Of the 16 patients whose LVAD flow was reduced, 14 (87.5\%) experienced resolution of ND (Table 3). Of 3 patients whose LVAD settings were not changed, 2 spontaneously recovered and 1 continued to deteriorate. In the 2 patients who recovered, the CI was noted to decrease from the time of the event from 2.75 to $2.4 \mathrm{~L} \cdot \mathrm{min}^{-1} \cdot \mathrm{m}^{-2}$, whereas in the patient whose condition continued to deteriorate, $\mathrm{CI}$ remained unchanged at $1.9 \mathrm{~L} \cdot \mathrm{min}^{-1} \cdot \mathrm{m}^{-2}$.

\section{DISCUSSION}

In a retrospective case series we found that of 69 patients with advanced heart failure who received the HeartMate XVE LVAD, 19 (27\%) had ND within 30 days after LVAD surgery. We also demonstrated a monotonic relationship between the probability of postoperative ND and the increase in CI after LVAD implantation. Our results therefore suggest an association between ND and an excessive increase in systemic blood flow. At the time of the neurologic event, the CI in nearly $80 \%$ of affected patients increased by greater than $50 \%$ from preoperative baseline values. The temporal association between decrease of flow and 


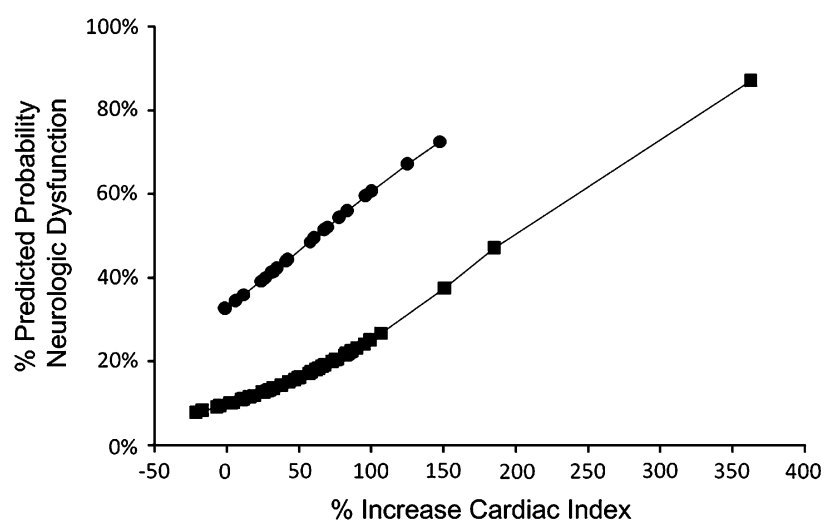

Previous coronary by-pass surgery $(n=21)$ No coronary by-pass surgery $(n=48)$

FIGURE 2. Relationship between the increase of cardiac index after left ventricular assist device implantation and the predicted probability of postoperative neurologic dysfunction depicted by the history of previous coronary bypass surgery.

resolution or improvement of symptoms suggests that ND might be a potentially preventable or perhaps reversible condition if related to cerebral hyperperfusion.

Although there are no published data quantifying cerebral flow in patients with advanced heart failure referred for LVAD implantation, it is likely that cerebral perfusion is severely impaired in this cohort given previous data showing abnormal cerebral blood flow in patients with heart failure. $^{12,13}$ Results of recent studies with intracranial Doppler studies $^{14}$ and radionuclide angiographic analysis ${ }^{15}$ have consistently demonstrated that in an ambulatory population of patients with New York Heart Association class III/IV heart failure, the baseline cerebral blood flow was reduced by at least $19 \%$ to $31 \%$, thus contributing to the frequently seen cognitive impairment in these patients. ${ }^{16}$

Because cerebral flow in LVAD candidates is probably markedly impaired, it is possible that an abrupt restoration of normal cardiac output after device implantation could overwhelm cerebral autoregulation, which is similar to that described in patients with severe internal carotid artery stenosis who had ND after carotid artery endarterectomy or angioplasty. ${ }^{9,17,18}$ Those patients' symptoms ranged from mild cognitive impairment to life-threatening intracranial hemorrhage, brain edema, and coma. The mechanism thought responsible for their complications was failure of the severely hypoperfused vasculature to respond to the rapidly increasing perfusion pressures. ${ }^{19,20}$

The abrupt restoration of high-output state after LVAD surgery and the accompanying postoperative ND might therefore constitute a model of whole-brain cerebral hyperperfusion. Our study showed that restoration of normal systemic flow after LVAD implantation in patients with severe heart failure was associated with a mean $42 \%$ increase in CI.

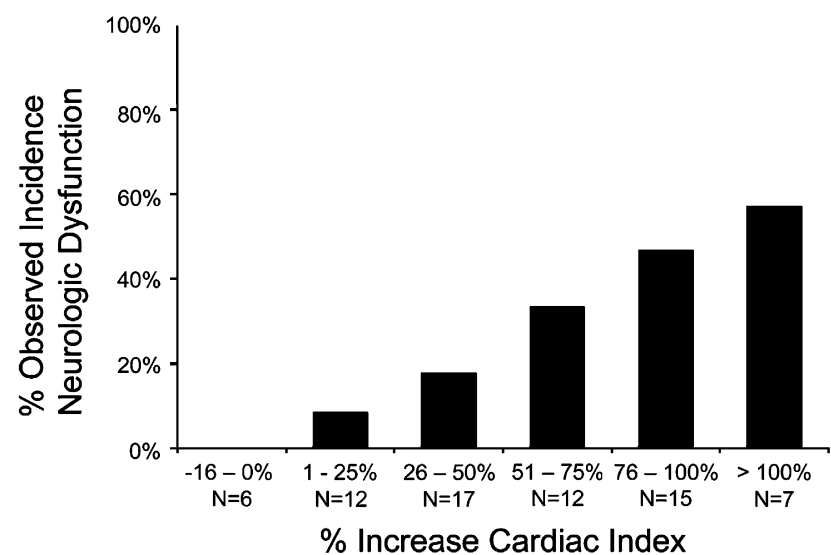

FIGURE 3. Observed incidence of postoperative neurologic dysfunction depicted by the increase in cardiac index after left ventricular assist device implantation.

Interestingly, the systemic blood pressure did not significantly increase with the increase in CI, and MAP was not a risk factor for ND, suggesting the probable primary role of systemic blood flow rather than blood pressure in this condition.

Most of the postoperative neurologic complications in the 19 affected patients were mild and included nonfocal symptoms involving patient cognition and affect: 12 had confusion or encephalopathy, and 2 became temporarily blind. Our results are consistent with those of studies of patients who had cerebral reperfusion syndrome after carotid endarterectomy or angioplasty, ${ }^{9,17,18}$ most of whom had nonfocal symptoms consistent with perfusion abnormalities in border zones.

It is important to note, however, that brain hyperperfusion can be a potentially life-threatening condition. Devastating complications with excessive LVAD flow, including severe brain edema and hemorrhage, were previously reported by Boyle and coworkers ${ }^{21}$ in 3 patients receiving HeartMate VE LVADs at our institution. At the time of the neurologic event, LVAD flow in all 3 of these patients was set at greater than $5 \mathrm{~L} / \mathrm{min}$. In our current study 6 patients had severe symptoms, including coma $(\mathrm{n}=2)$ and hemiparesis or hemiplegia $(n=4)$. In 5 of those patients, LVAD flow exceeded the $50 \%$ increase in CI from the preoperative baseline values; only 1 patient was found to have had an embolic stroke in imaging studies.

Of note, the term cerebral hyperperfusion syndrome has also been applied to conditions that involve an excessive increase in cerebral flow in the course of hypertensive encephalopathy ${ }^{20}$ or posterior reversible encephalopathy syndrome (PRES), which can develop in the presence of neurotoxins, such as cyclosporine. ${ }^{22}$ In our study, however, we found no significant association between systemic blood pressure and ND. There is one case of ND in a child after LVAD implantation in which researchers attributed symptoms to 

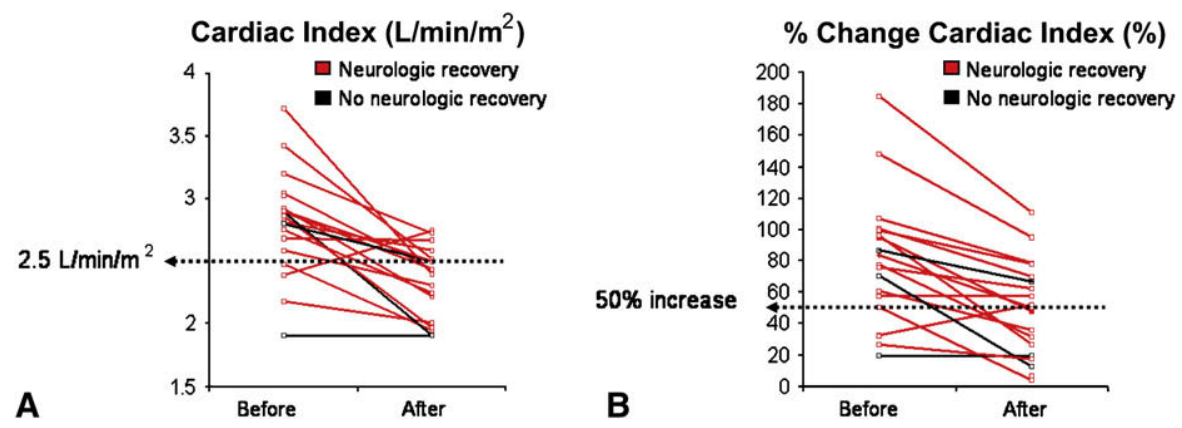

FIGURE 4. The reduction, before and after the neurologic event, in cardiac index (A) and percentage increase in cardiac index (B) in the 19 patients with neurologic dysfunction is shown. The red line indicates patients whose neurologic dysfunction improved or resolved after systemic flow was reduced. Black line, Patients whose neurologic dysfunction did not improve.

$\mathrm{PRES}^{23}$; of our 13 patients receiving LVADs who underwent CT analysis of the head, however, none were found to have radiographic evidence of PRES. Thus our results point to restoration of normal blood flow in severely hypoperfused circulation as the most likely mechanism contributing to ND.

It is important to note that advanced age and increased atherosclerosis burden might render these patients particularly vulnerable to the added effects of excess flow after LVAD insertion. Increased turbulence in the ascending aorta and aortic arch might increase atheroembolic risk, and previously unrecognized intracranial vascular disease might, in the setting of high flow, cause ND because of worsening of pre-existing, small-vessel, silent infarction or microhemorrhage. We show that LVAD recipients with coronary atherosclerosis and previous coronary bypass surgery were at substantially higher risk of postoperative ND. History of coronary bypass surgery was an independent risk factor for ND from that conferred by increased CI, suggesting that device flow might need to be titrated with greater scrutiny in older patients with coexisting atherosclerotic disease.

\section{LIMITATIONS}

Our study has several limitations. First, because of its retrospective nature, transcranial Doppler and brain perfusion studies were not performed after LVAD implantation, and therefore we have no direct measurements of cerebral blood flow at the time of ND. We made the general assumption that the device-generated systemic flow was an accurate measure of cardiac output. We were not able to explain what accounted for the excessive increase of systemic flow at the time of the neurologic event. Most of the patients with ND had their devices set in an automatic mode, which theoretically should adjust stroke volume to sensed inflow and maintain the systemic flow unchanged. The role of device-generated increase of pressure over time $(\Delta \mathrm{p} / \Delta \mathrm{t})$ was not studied. This study only examined recipients of HeartMate XVE LVADs, and these results might not apply to recipients of other devices.

Second, most symptomatic patients did not have formal neurologic evaluation, and no specific neuropsychological tests were given to determine which of the spectrum of symptoms improved best or most rapidly with reduction of device flow. Imaging was limited to CT scanning of the head because the presence of the LVAD excluded use of more sensitive radiographic measurement, such as magnetic resonance imaging. The CT scanning was done acutely, perhaps limiting its sensitivity to detect ischemic stroke. To avoid bias associated with a nonuniform workup of ND, we included in this study all patients with neurologic complications, regardless of the results of subsequent clinical evaluation with imaging or laboratory tests. Thus the strength of the relationship between ND and a change in CI might be underestimated.

Third, although postoperative vasodilation might be an important contributor to cerebral hyperperfusion and autonomic failure, the role of the postoperative use of vasodilators, such as angiotensin-converting enzyme inhibitors, nitrates, milrinone, inhaled nitric oxide, and infection, bleeding, and vasodilation syndrome, which are often seen in the postoperative period after LVAD implantation, were not studied. We were also not able to assess in all patients systemic vascular resistance to explain whether high device flow was related to systemic vasodilation. We also did not account for other metabolic disarrangements, which could contribute to encephalopathy.

Finally, 2 of 3 patients whose LVAD flow was not decreased did demonstrate spontaneous resolution of their $\mathrm{ND}$, and therefore it is possible that some of ND syndromes in the 16 patients whose LVAD output was decreased might have also resolved. It is also not yet known whether patients who demonstrated ND, even transiently, have different longterm outcomes either on the device or after heart transplantation.

\section{CONCLUSION}

In this retrospective series we found an association between an increase of blood flow after LVAD implantation and the development of ND. The manner in which cerebral hyperperfusion can contribute to postoperative ND in LVAD recipients could not be definitively explained 
in this observational study. Because cerebral hyperperfusion might be a potentially preventable and/or reversible condition, prospective studies, including direct measurements of cerebral blood flow and formal neurologic and neurocognitive evaluation, are necessary to better understand the role of cerebral hyperperfusion in ND after LVAD implantation.

\section{References}

1. Deng MC, Edwards LB, Hertz MI, Rowe AW, Keck BM, Kormos R, et al. Mechanical circulatory support device database of the International Society for Heart and Lung Transplantation: third annual report-2005. J Heart Lung Transplant. 2005;24:1182-7.

2. Pae WE, Connell JM, Boehmer JP, Korfer R, El-Banayosy A, Hetzer R, et al. Neurologic events with a totally implantable left ventricular assist device: European LionHeart Clinical Utility Baseline Study (CUBS). J Heart Lung Transplant. 2007;26:1-8.

3. Morgan JA, John R, Rao V, Weinberg AD, Lee BJ, Mazzeo PA, et al. Bridging to transplant with the HeartMate left ventricular assist device: the Columbia Presbyterian 12-year experience. J Thorac Cardiovasc Surg. 2004;127:1309-16.

4. Portner PM, Jansen PG, Oyer PE, Wheeldon DR, Ramasamy N. Improved outcomes with an implantable left ventricular assist system: a multicenter study. Ann Thorac Surg. 2001;71:205-9.

5. Ganushchak YM, Fransen EJ, Visser C, Do Jong DS, Maessen JG. Neurological complications after coronary artery bypass grafting related to the performance of cardiopulmonary bypass. Chest. 2004;125:2196-205.

6. Piccione W Jr. Left ventricular assist device implantation: short- and long-term surgical complications. J Heart Lung Transplant. 2000;19(suppl):S89-94.

7. Moazami N, Roberts K, Argenziano M, Catanese K, Mohr JP, Rose EA, et al. Asymptomatic microembolism in patients with long-term ventricular assist support. ASAIO J. 1997;43:177-80.

8. Lazar RM, Shapiro PA, Jaski BE, Parides MK, Bourge RC, Watson JT, et al. Neurological events during long-term mechanical circulatory support for heart failure: the Randomized Evaluation of Mechanical Assistance for the Treatment of Congestive Heart Failure (REMATCH) experience. Circulation. 2004;109:2423-7.
9. Van Mook WN, Rennenberg RJ, Schurink FW, van Oostenbrugge RJ, Mess WH, Hofman PA, et al. Cerebral hyperperfusion syndrome. Lancet Neurol. 2005;4: 877-88

10. Cox DR. Regression models and life tables. J R Stat Soc. 1972;B34:187.

11. Hosmer DW, Lemeshow S. Applied logistic regression. New York: John Wiley \& Sons; 1989.

12. Rajagopalan B, Raine AEG, Cooper R, Ledingham JGG. Changes in cerebral blood flow in patients with severe congestive cardiac failure before and after captopril treatment. Am J Med. 1984;76:86-90.

13. Paulson OB, Jarden JO, Gotfredsen J, Vorstrup S. Cerebral blood flow in patient with congestive heart failure treated with captopril. Am J Med. 1984;76:91-5.

14. Gruhn N, Larsen FS, Boesgaard S, Knudsen GM, Mortensen SA, Thomsen G, et al. Cerebral blood flow in patients with chronic heart failure before and after heart transplantation. Stroke. 2001;32:2530-3.

15. Choi BR, Kim JS, Yang YJ, Park KM, Lee CW, Kim YH, et al. Factors associated with decreased cerebral blood flow in congestive heart failure secondary to idiopathic dilated cardiomyopathy. Am J Cardiol. 2006;97:1365-9.

16. Pullicino PM, Hart J. Cognitive impairment in congestive heart failure?: embolism vs hypoperfusion. Neurology. 2001;57:1945-6.

17. Ogasawara K, Yamadate K, Kobayashi M, Fukada T, Yoshida K, Terasaki K, et al. Postoperative cerebral hyperperfusion associated with impaired cognitive function in patients undergoing carotid endarterectomy. J Neurosurg. 2005;102:38-44.

18. Fujimoto S, Toyoda K, Inoue T, Hirai Y, Uwatoko T, Kishikawa K, et al. Diagnostic impact of transcranial color-coded real-time sonography with echo contras agents for hyperperfusion syndrome after carotid endarterectomy. Stroke. 2004 35:1852-6.

19. Beausang-Linder M, Bill A. Cerebral circulation in acute arterial hypertensionprotective effects of sympathetic nervous activity. Acta Physiol Scand. 1981;111 193-9.

20. Gardner CJ, Lee K. Hyperperfusion syndromes: insight into the pathophysiology and treatment of hypertensive encephalopathy. CNS Spectr. 2007;12:35-42.

21. Boyle AJ, Park SJ, Colvin-Adams MM, Liao KK, Ormaza SM, Thompson JM, et al. Cerebral hyperperfusion syndrome following LVAD implantation [abstract]. J Heart Lung Transplant. 2003;22(suppl):S203.

22. Stott VL, Hurrell MA, Anderson TJ. Reversible posterior leukoencephalopathy syndrome: a misnomer reviewed. Intern Med J. 2005;35:83-90.

23. Williams LR, Darragh RK, Sokol DK, Edwards-Brown M, Turrentine MW. Reversible posterior leukoencephalopathy syndrome associated with left ventricular assist device. Transplant Proc. 2005;37:4513-4. 